\title{
Prognostic value of the KRAS G12V mutation in 841 surgically resected Caucasian lung adenocarcinoma cases
}

\author{
Stéphane Renaud ${ }^{1,2}$, Pierre-Emmanuel Falcoz ${ }^{\star 1}$, Mickaël Schaëffer $^{3}$, Dominique Guenot ${ }^{2}$, Benoit Romain ${ }^{2,4}$, \\ Anne Olland ${ }^{1}$, Jérémie Reeb ${ }^{1}$, Nicola Santelmo ${ }^{1}$, Marie-Pierre Chenard ${ }^{5}$, Michèle Legrain ${ }^{6}$, \\ Anne-Claire Voegeli ${ }^{6}$, Michèle Beau-Faller ${ }^{2,6}$ and Gilbert Massard ${ }^{1}$ \\ ${ }^{1}$ Department of Thoracic Surgery, Strasbourg University Hospital, Nouvel Hôpital Civil, 67000 Strasbourg, France; ${ }^{2}$ Reasearch Unit \\ EA3430: Tumoral Progression and Micro-environment, Translational and Epidemiological Approaches, Translational Medicine \\ Federation, Strasbourg University. 67000 Strasbourg, France; ${ }^{3}$ Department of Biostatistics, Strasbourg University Hospital. 67000 \\ Strasbourg, France; ${ }^{4}$ Department of General and Digestive Surgery, Strasbourg University Hospital, Hôpital de Hautepierre, \\ Strasbourg, France; ${ }^{5}$ Department of Pathology, Strasbourg University Hospital. 67000 Strasbourg, France and ${ }^{6}$ Department of \\ Molecular Biology, Oncobiology Laboratory, Regional Institute of Cancer Strasbourg University Hospital, Hôpital de Hautepierre, \\ Strasbourg, France
}

Background: Identifying patients who will experience lung cancer recurrence after surgery remains a challenge. We aimed to evaluate whether mutant forms of epidermal growth factor receptor (EGFR) and Kirsten rat sarcoma viral oncogene homolog (KRAS) (mEGFR and mKRAS) are useful biomarkers in resected non-small cell lung cancer (NSCLC).

Methods: We retrospectively reviewed data from 841 patients who underwent surgery and molecular testing for NSCLC between 2007 and 2012.

Results: mEGFR was observed in 103 patients (12.2\%), and mKRAS in 265 (31.5\%). The median overall survival (OS) and time to recurrence (TTR) were significantly lower for mKRAS (OS: 43 months; TTR: 19 months) compared with mEGFR (OS: 67 months; TTR: 24 months) and wild-type patients (OS: 55 months; disease-free survival (DFS): 24 months). Patients with KRAS G12V exhibited worse OS and TTR compared with the entire cohort (OS: KRAS G12V: 26 months vs Cohort: 60 months; DFS: KRAS G12V: 15 months vs Cohort: 24 months). These results were confirmed using multivariate analyses (non-G12V status, hazard ratio (HR): 0.43 (confidence interval: 0.28-0.65), $P<0.0001$ for OS; HR: 0.67 (0.48-0.92), $P=0.01$ for TTR). Risk of recurrence was significantly lower for non-KRAS G12V (HR: 0.01, (0.001-0.08), $P<0.0001)$.

Conclusions: mKRAS and mEGFR may predict survival and recurrence in early stages of NSCLC. Patients with KRAS G12V exhibited worse OS and higher recurrence incidences.

Lung cancer is the leading cause of cancer-related mortality worldwide. Non-small cell lung cancer (NSCLC) accounts for $\sim 80 \%$ of all lung cancer cases (Jemal et al, 2011). The 5-yearoverall survival (OS) rate across all stages is $<15 \%$ (Bossard et al, 2007). Even stage IA and IB NSCLC patients, for whom surgery is the cornerstone of treatment, exhibit a relatively poor prognosis, with $27 \%$ to $42 \%$ of these patients, respectively, dying within 5 years (Izar et al, 2014), primarily due to recurrence. The identification of patients who may experience recurrence after surgery remains a challenge. The TNM staging indicates the level

*Correspondence: Professor P-E Falcoz; E-mail: pierre-emmanuel.falcoz@wanadoo.fr

Received 13 May 2015; revised 23 July 2015; accepted 14 August 2015; published online 15 September 2015

(c) 2015 Cancer Research UK. All rights reserved 0007-0920/15 
of disease progression and the malignant potential of primary lung cancer. However, even patients with disease at the same stage exhibit wide variations in their incidence of recurrence after curative resection. Consequently, the current TNM staging based on clinical and pathological findings may have reached the limits of its usefulness (Uramoto and Tanaka, 2014).

Recent years have witnessed an increased understanding of the molecular alterations in tumours. Notably, NSCLC classification shifted from histological subtypes towards molecular oncogenic alterations. The main genomic alterations observed in NSCLC adenocarcinomas are epidermal growth factor receptor (EGFR) mutations, which occur in $10-20 \%$ of Caucasian patients, $V$-Ki-ras2 Kirsten rat sarcoma viral oncogene homolog (KRAS) oncogene, which is observed in $20-35 \%$ of patients, and anaplastic large cell lymphoma (ALK) gene fusion, which is observed in 5\% of patients (Zhang et al, 2014).

The published data on the prognostic value of these mutations in resected NSCLC are contradictory. A recent meta-analysis concluded an absence of impact of EGFR mutation on OS and disease-free survival (DFS) in resected NSCLC (Zhang et al, 2014). However, the study included a heterogeneous set of patients (primarily Asian) whose diagnoses ranged from stages I to IIIB, limiting the interpretation of these results. Another meta-analysis indicated a reduced OS in cases of KRAS mutation (Meng et al, 2013). However, different methods of KRAS mutation detection and treatment regimens were considered. Recent publications have demonstrated that different KRAS mutations may be classified into KRAS-dependent and KRAS-independent groups (Singh et al, 2009) and that the type of amino-acid substitution leads to differential binding affinity for downstream effector molecules. These results suggest that specific amino-acid substitutions are associated with different outcomes. However, the clinical data on resected lung NSCLC are poor and contradictory (Izar et al, 2014; Nadal et al, 2014).

We evaluate the prognostic value of EGFR mutations ( $m E G F R$ ) and KRAS mutations ( $m K R A S)$ with regard to specific amino-acid substitutions in 841 surgically treated French patients.

\section{PATIENTS AND METHODS}

The ethics committee of the French Society of Thoracic and Cardiovascular Surgeons approved this study (approval number: 2015-5-13-10-21-57-ReSt).

We retrospectively reviewed the data from 1971 patients who underwent molecular testing for KRAS and EGFR between January 2007 and December 2012 at the Molecular Biology Department of Strasbourg University Hospital (Strasbourg, France). Our study included 841 patients who underwent surgical resection with curative intent.

Molecular analysis. Samples were obtained from primary lung tumours. DNA was extracted from formalin-fixed paraffinembedded tumour samples, and EGFR/KRAS analysis was performed at exons 18-21 for EGFR and codon 12 out of 13 for KRAS, as previously described.(Beau-Faller et al, 2009; Beau-Faller et al, 2013) Wild-type (WT) patients were defined as patients who harboured no mEGFR or mKRAS. More recently, patient samples were tested for BRAF, PI3KCA, HER2 and ALK mutations.

Covariates and data collection. Baseline patient characteristics were collected, including age, sex, smoking history, neo-adjuvant and adjuvant treatment. The Charlson comorbidity index (CCI) was calculated for each patient. We grouped patients into the following established categories according to their total score: (Charlson et al, 1987) 0 (no comorbidity); 1-2 (average); 3-4 (moderate); and $\geq 5$ (severe). Smoking status was characterised as never a smoker, $<100$ cigarettes in their lifetime, a former smoker, quit $>1$ year before diagnosis and a current smoker with an ongoing smoking habit or who quit $<1$ year before diagnosis.

Pre-operative staging was performed using computed tomography (CT) scans of the chest, brain and upper abdomen, coupled with whole-body 18-fluorodeoxyglucose positron emission tomography and fibre optic bronchoscopy. Treatment decisions were made by a multidisciplinary board in the presence of a certified thoracic surgeon and a certified onco-pneumologist and radiation oncologist. Neo-adjuvant treatment consisted of chemotherapy either alone or in combination with radiation therapy (RT). All chemotherapy regimens were platinum-based. Some patients were referred to our facility by physicians from different centres. Therefore, no uniform protocol of neo-adjuvant therapy was used. Tumour stage was categorised according to the American Joint Committee on Cancer Staging Manual version 7. Dates and types of surgeries were recorded. Appropriate anatomical resections and systematic radical mediastinal lymphadenectomy were performed in accordance with the recommendations of the French Society of Thoracic and Cardiovascular surgeons (Thomas et al, 2008). Histopathological baseline characteristics, namely, angioinvasion, R0/R1/R2 and the number of N2 stations involved, were included. Skip metastases were defined as N2 involvement without N1. Microscopic N2 was defined as nodal metastases ranging from 0.2 to $2 \mathrm{~mm}$. Adjuvant chemotherapy consisted of platinum-based treatments, and RT was performed after CT-based three-dimensional treatment planning with a linear accelerator. The target volume included the area of loco-regional lymph nodes plus a margin of $2 \mathrm{~cm}$. The dose per fraction was $2 \mathrm{Gray}(\mathrm{Gy})$, given once daily, 5 days per week, up to a total dose of 60 Gy for R0 patients without extracapsular spread (ECS) or 66 Gy in cases of incomplete resection and/or ECS.

Patients were assessed for local and distant recurrence (DR), TTR and OS. The date of recurrence was defined as the first radiographic evidence of cancer relapse on imaging and/or pathological tumour evidence on biopsy. TKI was administered as a first-line treatment for recurrence in EGFR-mutated patients. The TTR was defined as time from surgery until the first diagnosis of recurrence on imaging or biopsy specimens. OS was defined as the time elapsed between surgery and either death or the last follow-up.

Statistical analyses. IBM SPSS (Armonk, NY, USA) v.20 was used for statistical analyses. Comparisons between groups were performed using $\chi^{2}$, medians, or Fisher's or Student's $t$-tests where appropriate. Correlations between qualitative variables were assessed using Cramer's V. The prognostic influence of variables on OS and DFS was assessed using the log-rank test and Cox proportional hazards models, and the influence of each variable on recurrence was assessed using a step-wise binary logistic regression. All tests were two-sided, and variables were considered significant for $P$ values $<0.05$. All variables with $P$ values $<0.2$ were tested in multivariate analyses.

\section{RESULTS}

Population characteristics. Our population was primarily male $(61.9 \%)$. The mean age at the time of diagnosis was 63.39 years $( \pm 11.52)$. The median follow-up time was 39 months (min, 8; max, 80). Molecular analyses revealed 265 mKRAS patients (31.5\%), 103 mEGFR patients (12.2\%) and $473 \mathrm{WT}$ patients (56.3\%). mEGFR status correlated with female sex (Cramer's V: $0.26, P<0.0001$ ) and non-smoking status (Cramer's V: 0.64, $P<0.0001)$, and $m K R A S$ status correlated with smoking status (Cramer's V: $0.1, P=0.02$ ). Significantly more skip $\mathrm{N}$ and microscopic $\mathrm{N}$ types were observed in $m E G F R$ patients $(P<0.0001$ for both). $m K R A S$ patients exhibited significantly more $\mathrm{pN}+(P<0.0001)$, involvement of two N2 stations 
$(P=0.004)$, angioinvasion $(P<0.0001)$ and the use of adjuvant treatment. There were more pT4 in WT patients $(P=0.002)$. There were no differences in age, $\mathrm{R} 0$ resection, neo-adjuvant treatment, type of resection and CCI score among the groups. Table 1 shows the sampled data.

Molecular data. Analyses of the EGFR mutations revealed 9 exon 18 mutations (five G791C, c.2155G > T; two G719A, c.2156G > C; and two G719S, c.2155G $>$ A), 45 exon 19 deletions, 17 exon 20 mutations (eight G796S, c.2386G- > A; seven S768I, c.2303G > T; and two VT65A, c.2294T > C), 34 exon 21 mutations (30 L858R, c. $2573 \mathrm{~T}>\mathrm{G}$; one R831C, c.2491C > T; two L861Q, c.2582T > A; and one G824V, c.2471G $>\mathrm{T}$ ), one exon 19 deletion and $790 \mathrm{M}$ exon 20 mutations, and one L858R exon 21 mutation with a
T790M exon 20 mutation. Analyses of mKRAS codon 12 transversions revealed 150 G12C (c.34G $>$ T, p.Gly12Cys), 90 G12V (c.35G $>$ T, p.Gly12Val), three G12A (c.35G $>$ C, p.Gly12Ala) and one G12R (c.34G > C, p.Gly12Arg). Analyses of mKRAS codon 12 transitions revealed nine G12D (c.35G $>$ A, p.Gly12Asp) and six G12S (c.34G >A, p.Gly12Ser). There were 10 G13C transversions (c.37G $>$ T, p.Gly13Cys) and one G13D transition (c.38G > A, p.Gly13Asp) on codon 13. Analyses of 171 patients $(20.3 \%)$ tested for HER2, PIK3CA and BRAF revealed a single patient who harboured a HER2 exon 20 insertion (0.6\%) and three (1.7\%) patients with a PIK3CA mutation (c.3140A $>\mathrm{G}$, H1047R). No BRAF mutations were noted. One $(6.2 \%)$ of the 16 patients tested harboured an $A L K$ fusion. All of these mutations were mutually exclusive from one another.

\begin{tabular}{|c|c|c|c|c|}
\hline & mKRAS & mEGFR & WT KRAS/EGFR & $P$ value \\
\hline Total $N$ & $265(31.5)$ & $103(12.2)$ & $473(56.3)$ & \\
\hline Male & $174(65.7)$ & $29(28.4)$ & $318(67.2)$ & $<0.0001$ \\
\hline Mean age $(y)$ & $63.26 \pm 10.07$ & $63.67 \pm 14.23$ & $63.39 \pm 11.65$ & 0.95 \\
\hline pT & & & & 0.002 \\
\hline $\begin{array}{l}1 \\
2 \\
3 \\
4\end{array}$ & $\begin{array}{r}59(22.3) \\
129(48.7) \\
66(24.9) \\
11(4.2)\end{array}$ & $\begin{array}{c}22(21.4) \\
49(47.6) \\
30(29.1) \\
2(1.9)\end{array}$ & $\begin{array}{c}132(27.9) \\
173(36.6) \\
125(26.4) \\
43(9.1)\end{array}$ & \\
\hline $\mathrm{pN}+$ & $216(81.5)$ & $32(31.1)$ & $177(37.4)$ & $<0.0001$ \\
\hline $\mathrm{pN}$ & & & & $<0.0001$ \\
\hline $\begin{array}{l}\mathrm{pN} 0 \\
\mathrm{pN} 1 \\
\mathrm{pN} 2\end{array}$ & $\begin{array}{r}49(18.5) \\
156(58.9) \\
60(22.6)\end{array}$ & $\begin{array}{l}71(68.9) \\
15(14.6) \\
17(16.5)\end{array}$ & $\begin{array}{r}296(62.6) \\
84(17.8) \\
93(19.7)\end{array}$ & \\
\hline Skip N & $9(15)$ & $9(60)$ & 0 & $<0.0001$ \\
\hline Microscopic N & $20(9.3)$ & $17(53.1)$ & 0 & $<0.0001$ \\
\hline Number of N2 stations & & & & 0.004 \\
\hline $\begin{array}{l}1 \\
2\end{array}$ & $\begin{array}{l}37(61.7) \\
23(38.3)\end{array}$ & $\begin{array}{r}14(82.4) \\
3(17.6)\end{array}$ & $\begin{array}{l}78(84.8) \\
14(15.2)\end{array}$ & \\
\hline Smoking & & & & $<0.0001$ \\
\hline $\begin{array}{l}\text { Never } \\
\text { Past } \\
\text { Current }\end{array}$ & $\begin{array}{r}36(13.6) \\
101(38.1) \\
128(48.3)\end{array}$ & $\begin{aligned} 91 & (88.3) \\
6 & (5.8) \\
6 & (5.8)\end{aligned}$ & $\begin{array}{c}43(9.1) \\
210(44.4) \\
220(46.5)\end{array}$ & \\
\hline RO resection & $263(99.2)$ & $101(98.1)$ & $464(98.1)$ & 0.45 \\
\hline Angioinvasion & $155(58.5)$ & $17(16.5)$ & $157(33.2)$ & $<0.0001$ \\
\hline Neo-adjuvant treatment & $97(36.6)$ & $34(33)$ & $198(41.9)$ & 0.15 \\
\hline Type of neo-adjuvant treatment & & & & 0.15 \\
\hline $\begin{array}{l}\text { Chemo. } \\
\text { RT chemo. }\end{array}$ & $\begin{array}{l}74(76.3) \\
23(23.7)\end{array}$ & $\begin{array}{l}23(67.6) \\
11(32.4)\end{array}$ & $\begin{array}{r}160(80.8) \\
38(19.2)\end{array}$ & \\
\hline Type of resection & & & & 0.13 \\
\hline $\begin{array}{l}\text { Lobectomy } \\
\text { Bi-lobectomy } \\
\text { Pneumonectomy } \\
\text { Segmentectomy }\end{array}$ & $\begin{aligned} 243 & (91.7) \\
12 & (4.5) \\
1 & (0.4) \\
9 & (3.4)\end{aligned}$ & $\begin{array}{l}9(88.3) \\
4(3.9) \\
2(1.9) \\
6(5.8)\end{array}$ & $\begin{array}{l}403(85.2) \\
32(6.8) \\
14(3) \\
24(5.1)\end{array}$ & \\
\hline Adjuvant treatment & $217(81.9)$ & $34(33)$ & $183(38.7)$ & $<0.0001$ \\
\hline Type of adjuvant treatment & & & & $<0.0001$ \\
\hline $\begin{array}{l}\text { RT } \\
\text { Chemo. } \\
\text { RT chemo. }\end{array}$ & $\begin{array}{c}1(0.5) \\
194(89.4) \\
22(10.1)\end{array}$ & $\begin{array}{c}2(5.9) \\
32(94.1) \\
0\end{array}$ & $\begin{array}{r}6(3.3) \\
149(81.4) \\
28(15.3)\end{array}$ & \\
\hline $\mathrm{CCl}$ & & & & 0.45 \\
\hline $\begin{array}{l}0 \\
1 \\
2 \\
3 \\
\end{array}$ & $\begin{array}{l}22(8.3) \\
99(37.4) \\
72(27.2) \\
72(27.2) \\
\end{array}$ & $\begin{array}{l}13(12.6) \\
46(44.7) \\
21(20.4) \\
23(22.3) \\
\end{array}$ & $\begin{array}{r}54(11.4) \\
171(36.2) \\
120(25.4) \\
128(27.1) \\
\end{array}$ & \\
\hline
\end{tabular}



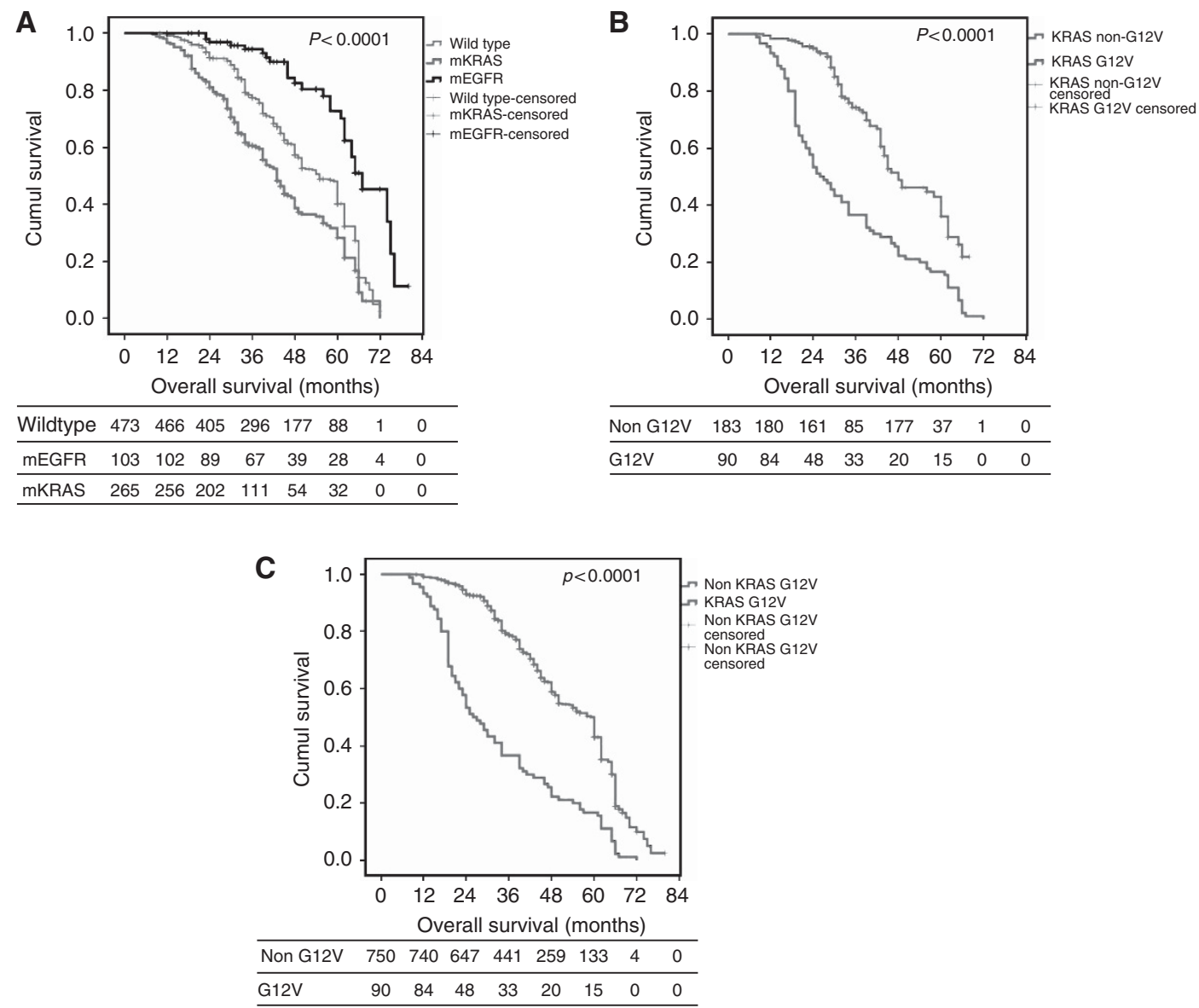

Figure 1. Kaplan-Meier overall survival. (A) According to the mutational status, (B) according to G12V vs other KRAS mutants, (C) according to KRAS G12V or non-KRAS G12V status.

Overall survival. The median OS was 49 months for the entire population, with 1-, 3-, 5- and 6-year OS rates of 98\%, 74\%, $41 \%$ and $12 \%$, respectively. Univariate analysis revealed that mutational status significantly influenced OS. The median OS was 55 months for WT patients (95\% confidence interval (CI)]: 51.42-58.57), which increased to 67 months for mEGFR patients (95\% CI: 59.43-74.57) and decreased to 43 months for $m K R A S$ patients (95\% CI: $39.53-46.47$ ), with 5 -y OS rates of $38 \%, 71 \%$ and $27 \%$, respectively $(P<0.0001$; Figure $1 \mathrm{~A})$. Because TKI was given as a first-line treatment in cases of recurrence to mEGFR, we evaluated whether the use of TKI was associated with better OS. However, there was no significant difference between patients who benefitted from TKI ( $n=37$, median OS: 58 months (95\% CI: 46-64)) and those who did not (median OS: 54 months (95\% CI: $50-60), P=0.89)$. The $m E G F R$ type did not affect OS $(P=0.72)$. However, the type of $m K R A S$ significantly influenced OS $(P<0.0001)$. The median OS was not reached for G12D patients but did reach 62 months for G12R patients. The median OS decreased to 60 months for G12S patients, 49 months (95\% CI: 38.17-59.83) for G12C patients, 45 months (95\% CI: 21.68-68.32) for G13C patients, 39 months (95\% CI: 29.39-48.6) for G13D patients, 30 months (95\% CI: 15.59-44.4) for G12A patients and 26 months (95\% CI: 20.99-31.01) for G12V patients. We pooled $m K R A S$ patients into non-G12V and G12V groups because of the poor median OS for $\mathrm{G} 12 \mathrm{~V}$ patients. G12V patients had a significantly lower median OS than non-G12V patients (26 months 95\% CI: 20.99-31.01 vs 48 months 95\% CI: 38.9-57.1, respectively; $P<0.0001$; Figure 1B). After ensuring that the median OS of KRAS non-G12V (48 months 95\% CI: 38.9-57.1) was not significantly different from that of WT and mEGFR (60 months
95\% CI: 49.3-61.2, $P=0.12$ ), the median OS of the entire cohort (including mEGFR, WT and non-G12V KRAS patients) was compared with the median OS of KRAS G12V patients; accordingly, the median OS was significantly lower for KRAS G12V patients (G12V, 26 months, 95\% CI: 20.99-31.01 vs Cohort, 60 months, 95\% CI: 58.56-63.44, $P<0.0001$; Figure 1C). Univariate analyses revealed that gender $(P=0.003)$, nodal status $(P=0.001)$, pT $(P<0.0001)$, angioinvasion $(P<0.0001)$, smoking status $(P<0.0001)$, neo-adjuvant treatment $(P=0.002)$, type of neo-adjuvant treatment $(P=0.001)$, adjuvant treatment $(P=0.002)$, type of adjuvant treatment $(P=0.015)$, type of resection $(P<0.0001)$ and microscopic $\mathrm{N}(P=0.05)$ significantly influenced the median OS. However, multivariate analyses revealed that only KRAS G12V status (hazard ratio (HR): $2.1,95 \%$ CI: $1.31-3.37, P=0.002$ ) and the absence of angioinvasion (HR: 0.58, 95\% CI: 0.34-0.99, $P=0.05)$ remained independent prognostic factors. These data are compiled in Table 2. A correlation was observed between KRAS status and angioinvasion (58.8\% of angioinvasion for $m K R A S v s$ $30.1 \%$ for the rest of the population, Cramer's V: $0.28, P<0.0001$ ) and, in particular, with KRAS G12V (98.9\% of angioinvasion for KRAS G12V vs 38.8\% for KRAS non-G12V, Cramer's V: 0.58 , $P<0.0001)$. However, no further tests of interaction could be performed due to problems associated with separating the statistical data. Indeed, all G12V patients, except one, exhibited angioinvasion.

Time to recurrence. The median TTR for the entire cohort was 48 months, with corresponding 1-, 2-, 3- and 5-year TTR rates of $85 \%, 43 \%, 37 \%$ and $8 \%$, respectively. Univariate analyses revealed that mutational status significantly influenced TTR. The median 
Table 2. Uni- and multivariate analyses of overall survival (OS)

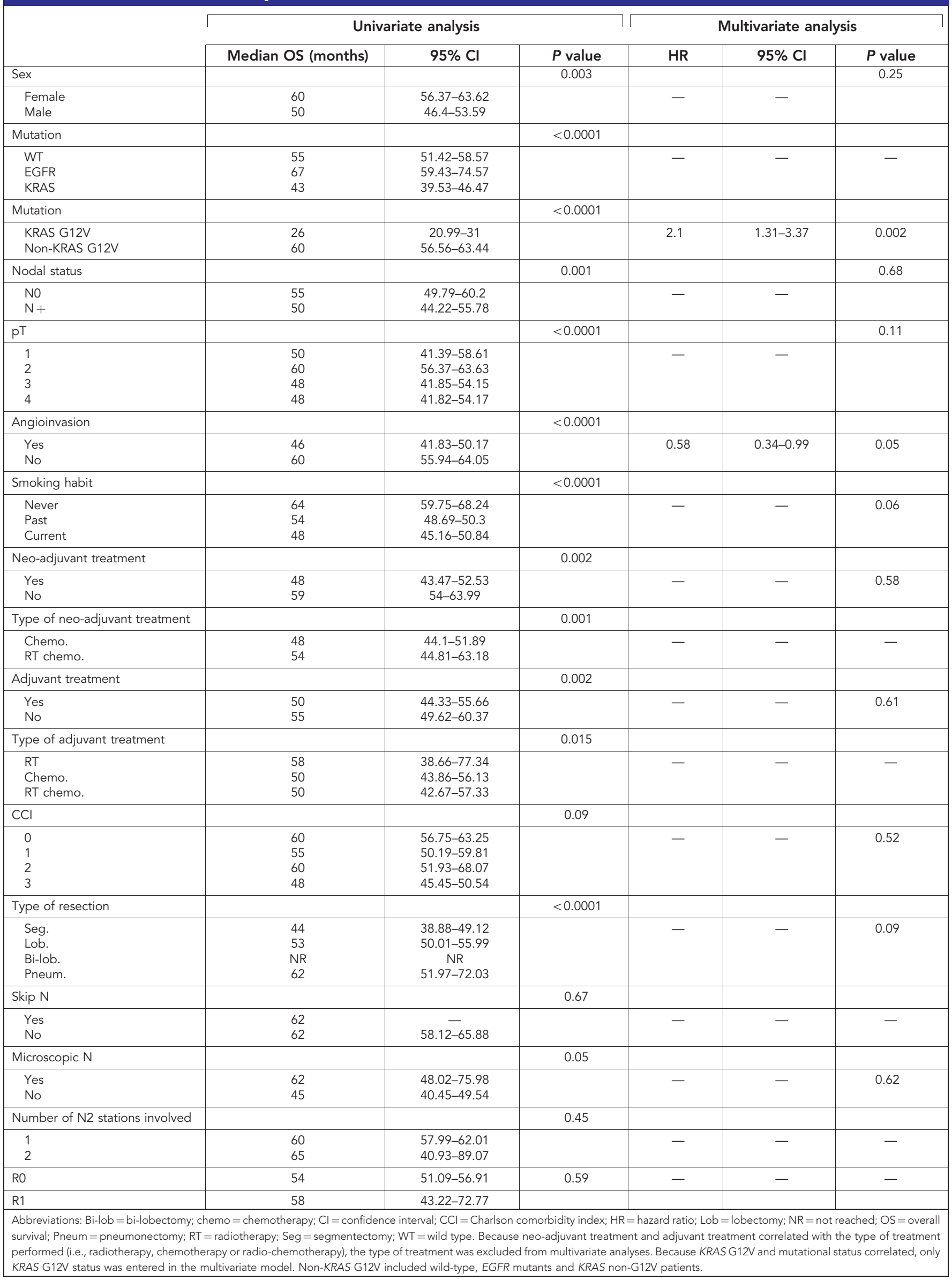


A

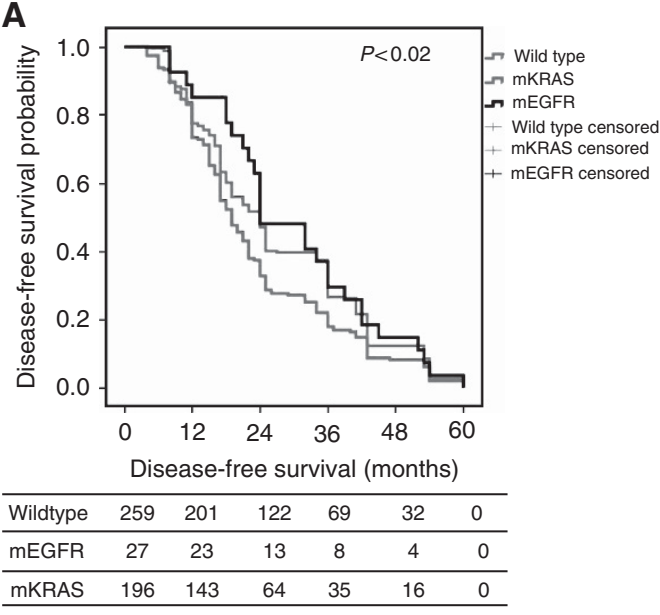

B

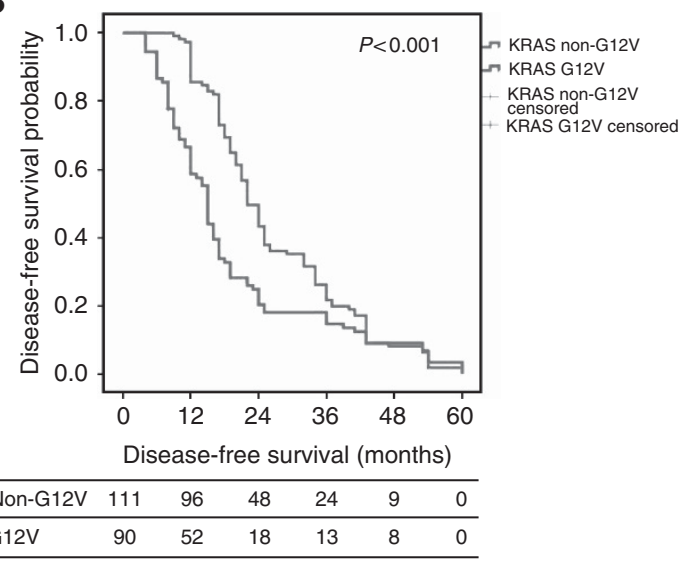

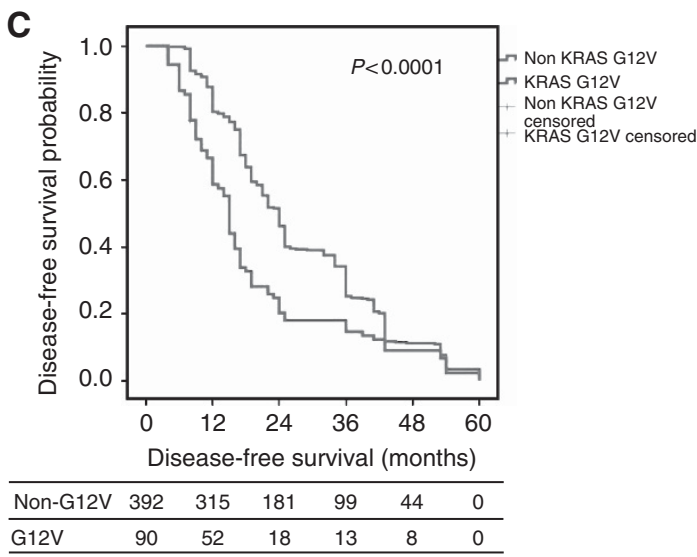

Figure 2. Kaplan-Meier recurrence-free survival. (A) According to the mutational status, (B) according to G12V vs other KRAS mutants, (C) according to KRAS G12V or non-KRAS G12V status.

TTR reached 24 months (95\% CI: 21.69-26.3) for WT patients and $m E G F R$ patients (95\% CI: 16.37-31.63), whereas it decreased to 19 months (95\% CI: 17.19-20.8) for $m K R A S$ patients $(P=0.01$; Figure 2A). The type of $m E G F R$ did not significantly influence TTR $(P=0.97)$, but the type of $m K R A S$ did significantly influence TTR. The TTR was 32 months (95\% CI: 7.99-56) for G12S patients, 24 months (95\% CI: 21.93-26.07) for G12C patients, 18 months (95\% CI: 7.22-28.78) for G12D patients, 17 months for G13D patients, 15 months (95\% CI: 14.08-15.91) for G13C and G12A patients, 14 months for G12R patients and 12 months for G12V patients (95\% CI: 5.53-18.47; $P=0.02$ ). KRAS G12V patients were compared with KRAS non-G12V patients. The median TTR was significantly lower for KRAS G12V patients compared with KRAS non-G12V patients (12 months, 95\% CI: 5.53-18.47 vs 22 months, 95\% CI: 19.93-24.07, respectively; $P=0.001$; Figure 2B). Comparison of the TTR of KRAS G12V patients to the entire cohort revealed that the median DFS was still significantly lower in KRAS G12V patients (G12V, 12 months, 95\% CI: 5.53-18.47 vs Cohort, 24 months, 95\% CI: 22.71-25.29, $P<0.0001$; Figure 2C). Univariate analysis revealed that angioinvasion $(P=0.01)$, neo-adjuvant treatment type $(P=0.04)$ and skip $\mathrm{N}(P=0.002)$ influenced TTR. However, multivariate analysis revealed that only the absence of KRAS G12V status remained an independent prognostic factor (HR: 0.67, 95\% CI: 0.48-0.92, $P=0.01)$. These data are compiled in Table 3.

Loco-regional and distant recurrence. At the end of the followup period, 481 patients (57.2\%) had experienced a local and/or distant recurrence (DR): 378 patients with loco-regional recurrence (44.9\%) and 281 patients with a DR (33.4\%). Univariate analyses revealed that mutation status influenced the risk of recurrence. Indeed, $54.8 \%$ of WT patients experienced recurrence $v s$ only $36.2 \%$ of $m E G F R$ and $74 \%$ of $m K R A S$ patients $(P<0.0001)$. The risk of recurrence was not significantly different according to the type of EGFR mutation $(P=0.67)$, but the type of KRAS mutation significantly influenced recurrence risk $(P<0.0001)$. Indeed, $89 \mathrm{G} 12 \mathrm{~V}$ patients (98.9\%), 9 G13C patients (90\%), one G12R patient $(100 \%)$, eight G13C patients $(88.9 \%)$, two G12A patients (66.7\%), $91 \mathrm{G} 12 \mathrm{C}$ patients (60.7\%), three G12S patients (50\%), four G12D patients $(44.4 \%)$ and one G13D patient $(25 \%)$ experienced recurrence. Comparison of KRAS G12V patients with KRAS nonG12V patients revealed that the risk of recurrence was significantly higher for the former (OR: 57.7, 95\% CI: 7.87-423.65, $P<0.0001$ ). The risk of recurrence was significantly higher for KRAS G12V patients compared with that of the entire cohort (OR: 81.5, 95\% CI: 11.3-588.06, $P<0.0001)$. Univariate analyses revealed that gender $(P=0.02)$, nodal status $(P<0.0001)$, pT $(P=0.01)$, angioinvasion $(P=0.0007)$, smoking habit $(P<0.0001)$, adjuvant treatment $(P<0.0001)$ and type of resection $(P<0.0001)$ influenced recurrence. However, multivariate analyses revealed that only non-KRAS G12V status remained an independent prognostic factor of recurrence (HR: 0.01, 95\% CI: 0.001-0.08, $P<0.0001$ ). These data are compiled in Table 4.

\section{DISCUSSION}

The prognostic and predictive values of $m E G F R$ in advanced stage NSCLC are clearly established, and mEGFR patients benefit from 
Table 3. Uni- and multivariate analyses of time to recurrence (TTR)

\begin{tabular}{|c|c|c|c|c|c|c|}
\hline & \multicolumn{3}{|c|}{ Univariate analysis } & \multicolumn{3}{|c|}{ Multivariate analysis } \\
\hline & Median OS (months) & $95 \% \mathrm{Cl}$ & $P$ value & HR & $95 \% \mathrm{Cl}$ & $P$ value \\
\hline Sex & & & 0.72 & & & \\
\hline $\begin{array}{l}\text { Female } \\
\text { Male }\end{array}$ & $\begin{array}{l}22 \\
21\end{array}$ & $\begin{array}{c}19.8-24.19 \\
18.71-23.29\end{array}$ & & - & - & - \\
\hline Mutation & & & 0.01 & & & \\
\hline $\begin{array}{l}\text { WT } \\
\text { EGFR } \\
\text { KRAS }\end{array}$ & $\begin{array}{l}24 \\
24 \\
19\end{array}$ & $\begin{array}{c}21.69-26.3 \\
16.37-31.63 \\
17.19-20.8\end{array}$ & & - & - & - \\
\hline Mutation & & & $<0.0001$ & & & \\
\hline $\begin{array}{l}\text { KRAS G12V } \\
\text { Non-KRAS G12V }\end{array}$ & $\begin{array}{l}15 \\
24\end{array}$ & $\begin{array}{l}13.46-16.54 \\
22.71-25.29\end{array}$ & & 0.67 & $0.48-0.92$ & 0.01 \\
\hline Nodal status & & & 0.77 & & & \\
\hline $\begin{array}{l}\mathrm{N} 0 \\
\mathrm{~N}+\end{array}$ & $\begin{array}{l}21 \\
22\end{array}$ & $\begin{array}{l}18.08-23.92 \\
19.97-24.03\end{array}$ & & - & - & - \\
\hline pT & & & 0.09 & & & \\
\hline $\begin{array}{l}1 \\
2 \\
3 \\
4\end{array}$ & $\begin{array}{l}21 \\
24 \\
19 \\
19\end{array}$ & $\begin{array}{c}18.3-23.7 \\
21.78-26.22 \\
15.64-22.36 \\
14-23.99\end{array}$ & & - & - & 0.08 \\
\hline Angioinvasion & & & 0.01 & & & \\
\hline $\begin{array}{l}\text { Yes } \\
\text { No }\end{array}$ & $\begin{array}{l}18 \\
24\end{array}$ & $\begin{array}{l}16.11-19.88 \\
22.56-25.44\end{array}$ & & - & - & 0.38 \\
\hline Smoking habit & & & 0.11 & & & \\
\hline $\begin{array}{l}\text { Never } \\
\text { Past } \\
\text { Current }\end{array}$ & $\begin{array}{l}24 \\
21 \\
21\end{array}$ & $\begin{array}{l}14.14-33.86 \\
17.24-24.76 \\
18.94-23.06\end{array}$ & & - & - & 0.59 \\
\hline Neo-adjuvant treatment & & & 0.26 & & & \\
\hline $\begin{array}{l}\text { Yes } \\
\text { No }\end{array}$ & $\begin{array}{l}21 \\
22\end{array}$ & $\begin{array}{l}18.32-23.68 \\
19.98-24.01\end{array}$ & & - & - & - \\
\hline Type of neo-adjuvant treatment & & & 0.04 & & & \\
\hline $\begin{array}{l}\text { Chemo. } \\
\text { RT chemo. }\end{array}$ & $\begin{array}{l}19 \\
25\end{array}$ & $\begin{array}{l}16.11-21.89 \\
20.97-29.02\end{array}$ & & - & - & 0.57 \\
\hline Adjuvant treatment & & & 0.9 & & & \\
\hline $\begin{array}{l}\text { Yes } \\
\text { No }\end{array}$ & $\begin{array}{l}22 \\
21\end{array}$ & $\begin{array}{l}19.59-24.41 \\
18.42-23.58\end{array}$ & & - & - & - \\
\hline Type of adjuvant treatment & & & 0.4 & & & \\
\hline $\begin{array}{l}\text { RT } \\
\text { Chemo. } \\
\text { RT chemo. }\end{array}$ & $\begin{array}{l}34 \\
21 \\
25\end{array}$ & $\begin{array}{c}21.99-46 \\
18.38-23.62 \\
19.37-30.63\end{array}$ & & - & - & - \\
\hline $\mathrm{CCl}$ & & & 0.56 & & & \\
\hline $\begin{array}{l}0 \\
1 \\
2 \\
3\end{array}$ & $\begin{array}{l}22 \\
24 \\
21 \\
19\end{array}$ & $\begin{array}{l}16.91-27.09 \\
21.88-26.12 \\
18.38-23.62 \\
15.95-22.05 \\
\end{array}$ & & - & - & - \\
\hline Type of resection & & & 0.34 & & & \\
\hline $\begin{array}{l}\text { Seg. } \\
\text { Lob. } \\
\text { Bi-lob. } \\
\text { Pneum. }\end{array}$ & $\begin{array}{l}24 \\
21 \\
17 \\
25\end{array}$ & $\begin{array}{l}15.84-32.15 \\
18.93-23.06 \\
18.06-31.94\end{array}$ & & - & - & - \\
\hline Skip N & & & 0.002 & & & \\
\hline $\begin{array}{l}\text { Yes } \\
\text { No }\end{array}$ & $\begin{array}{l}19 \\
25\end{array}$ & $\begin{array}{l}13.87-24.13 \\
16.05-33.94\end{array}$ & & - & - & 0.19 \\
\hline Microscopic N & & & 0.12 & & & \\
\hline $\begin{array}{l}\text { Yes } \\
\text { No }\end{array}$ & $\begin{array}{l}24 \\
18\end{array}$ & $\begin{array}{c}19.3-28.69 \\
16.16-19.83\end{array}$ & & - & - & 0.23 \\
\hline Number of N2 stations involved & & & 0.45 & & & \\
\hline $\begin{array}{l}1 \\
2\end{array}$ & $\begin{array}{l}25 \\
25\end{array}$ & $\begin{array}{l}21.78-28.22 \\
15.81-34.19\end{array}$ & & - & - & - \\
\hline RO & 21 & $19.38-22.62$ & 0.51 & - & - & - \\
\hline R1 & 34 & $7.7-60.29$ & & & & \\
\hline
\end{tabular}


Table 4. Uni- and multivariate analyses of recurrence

\begin{tabular}{|c|c|c|c|c|c|c|}
\hline & \multicolumn{3}{|c|}{ Univariate analysis } & \multicolumn{3}{|c|}{ Multivariate analysis } \\
\hline & $n(\%)$ & OR $(95 \% \mathrm{Cl})$ & $P$ value & HR & $95 \% \mathrm{Cl}$ & $P$ value \\
\hline Sex & & & 0.02 & & & \\
\hline $\begin{array}{l}\text { Female } \\
\text { Male }\end{array}$ & $\begin{array}{l}166(52) \\
316(60.7)\end{array}$ & $1.42(1.07-1.89)$ & & - & - & 0.21 \\
\hline Mutation & & & $<0.0001$ & & & \\
\hline $\begin{array}{l}\text { WT } \\
\text { EGFR } \\
\text { KRAS }\end{array}$ & $\begin{array}{c}259(54.8) \\
27(26.2) \\
196(74)\end{array}$ & - & & - & - & - \\
\hline Mutation & & & $<0.0001$ & & & \\
\hline $\begin{array}{l}\text { KRAS G12V } \\
\text { Non-KRAS G12V }\end{array}$ & $\begin{array}{r}89(98.9) \\
392(52.2)\end{array}$ & $81.5(11.3-588.06)$ & & 0.01 & $0.001-0.08$ & $<0.0001$ \\
\hline Nodal status & & & $<0.0001$ & & & \\
\hline $\begin{array}{l}\mathrm{NO} \\
\mathrm{N}+\end{array}$ & $\begin{array}{l}208(50) \\
274(64.5)\end{array}$ & $1.81(1.38-2.39)$ & & - & - & 0.53 \\
\hline pT & & & 0.01 & & & \\
\hline $\begin{array}{l}1 \\
2 \\
3 \\
4\end{array}$ & $\begin{array}{r}107(50.2) \\
211(60.1) \\
138(62.4) \\
26(46.4)\end{array}$ & & & - & - & 0.88 \\
\hline Angioinvasion & & & 0.007 & & & \\
\hline $\begin{array}{l}\text { Yes } \\
\text { No }\end{array}$ & $\begin{array}{l}208(63.2) \\
274(53.5)\end{array}$ & $1.49(1.12-1.98)$ & & - & - & 0.07 \\
\hline Smoking habit & & & $<0.0001$ & . & & \\
\hline $\begin{array}{l}\text { Never } \\
\text { Past } \\
\text { Current }\end{array}$ & $\begin{array}{c}68(40) \\
198(62.5) \\
216(61)\end{array}$ & & & - & - & 0.28 \\
\hline Neo-adjuvant treatment & & & 0.88 & 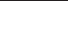 & & \\
\hline $\begin{array}{l}\text { Yes } \\
\text { No }\end{array}$ & $\begin{array}{l}187(38.8) \\
295(61.2)\end{array}$ & $0.97(0.73-1.28)$ & & - & - & - \\
\hline Type of neo-adjuvant treatment & & & 0.25 & - & & \\
\hline $\begin{array}{l}\text { Chemo. } \\
\text { RT chemo. }\end{array}$ & $\begin{array}{r}140(54.5) \\
47(65.3)\end{array}$ & $1.28(0.65-2.12)$ & & - & - & - \\
\hline Adjuvant treatment & & & $<0.0001$ & 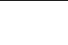 & & \\
\hline $\begin{array}{l}\text { Yes } \\
\text { No }\end{array}$ & $\begin{array}{l}280(64.5) \\
202(49.6)\end{array}$ & $1.84(1.4-2.43)$ & & - & - & 0.33 \\
\hline Type of adjuvant treatment & & & 0.33 & & & \\
\hline $\begin{array}{l}\text { RT } \\
\text { Chemo. } \\
\text { RT chemo. }\end{array}$ & $\begin{array}{r}6(66.7) \\
241(64.3) \\
33(66)\end{array}$ & - & & - & - & - \\
\hline Type of resection & & & $<0.0001$ & 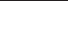 & & \\
\hline $\begin{array}{l}\text { Seg. } \\
\text { Lob. } \\
\text { Bi-lob. } \\
\text { Pneum. }\end{array}$ & $\begin{aligned} 23 & (59) \\
427 & (57.9) \\
1 & (5.9) \\
31 & (64.6)\end{aligned}$ & - & & - & - & 0.15 \\
\hline Skip N & & & 0.46 & & & \\
\hline $\begin{array}{l}\text { Yes } \\
\text { No }\end{array}$ & $\begin{array}{r}7(38.9) \\
30(52.6)\end{array}$ & $0.57(0.19-1.69)$ & & - & - & - \\
\hline Microscopic N & & & 0.9 & & & \\
\hline $\begin{array}{l}\text { Yes } \\
\text { No }\end{array}$ & $\begin{array}{r}23(62.2) \\
251(64.7)\end{array}$ & $0.89(0.45-1.8)$ & & - & - & - \\
\hline Number of N2 stations involved & & & 0.94 & . & & \\
\hline $\begin{array}{l}1 \\
2\end{array}$ & $\begin{array}{l}68(52.7) \\
22(55)\end{array}$ & $1.09(0.54-2.23)$ & & - & - & - \\
\hline RO & $473(57.1)$ & $1.69(0.52-5.23)$ & 0.55 & - & - & - \\
\hline R1 & $9(69.2)$ & & & & & \\
\hline
\end{tabular}


EGFR TKI treatment. However, $m K R A S$ is associated with resistance to these therapies. The impact of both mutations on OS and TTR in resected patients has not been well studied, and results are conflicting. Notably, only three previous studies directly compared EGFR, KRAS and WT patients (Marks et al, 2008; D'Angelo et al, 2012; Izar et al, 2014), and only two of these studies provided data on DFS (D’Angelo et al, 2012; Izar et al, 2014). Only two previous studies of small cohorts focused on the impact of KRAS-specific amino-acid substitutions on OS and DFS in resected NSCLC (Izar et al, 2014; Nadal et al, 2014), with conflicting results.

A recent meta-analysis of 3337 patients on the impact of $m E G F R$ on resected NSCLC demonstrated no significant impact of EGFR mutational status on DFS or OS (Zhang et al, 2014). However, only 6 out of the 22 studies included were based on nonAsian patients. Notably, the rates of $m E G F R$ were $>25 \%$ in 14 studies, although these values do not reflect the expected rate in a Caucasian population (approaching 10\%). Furthermore, only 12 studies provided data on DFS. These populations were also quite heterogeneous and included stages I to IIIA NSCLC with various NSCLC histologies and no uniform exon sequencing. All of these observations limit the interpretation of these previous results. We demonstrated that $m E G F R$ was associated with improved OS and TTR compared with $m K R A S$ and WT patients, which is consistent with previous publications (Marks et al, 2008; D’Angelo et al, 2012; Izar et al, 2014). Notably, we observed that TTR was significantly better in mEGFR than mKRAS patients. However, the median DFS was not significantly different between $m E G F R$ and WT patients. In our study, EGFR TKIs were systematically administered to $m E G F R$ patients in cases of recurrence. However, whether the benefit of improved OS in mEGFR patients can be attributed to TKIs rather than the mutational status itself is not known. Indeed, in our cohort, regardless of whether we observed a significant difference between patients who benefit from TKI, the use of TKI was very low (4.4\%), making it impossible to draw a firm conclusion with respect to its role. However D'Angelo et al (2012), showed that resected $m E G F R$ patients who received adjuvant TKI had a longer DFS compared with patients who did not (HR: 0.43 $(0.26-0.72), P=0.001)$. However, the improvements in OS in these patients were not significant (HR: $0.5(0.23-1.08), P=0.076)$. In their study, the patients who received adjuvant EGFR TKI had a higher disease stage and received significantly more chemotherapy before the initiation of TKI ( $45 \%$ vs $16 \%, P<0.001)$. However, in the absence of targeted therapy Marks et al (2008), nonetheless demonstrated that $m E G F R$ was associated with improved OS compared with WT and mKRAS patients; however, no data on DFS were provided. Their population study was also relatively small, including only $40 \mathrm{mEGFR}$ patients. Consequently, the introduction of TKIs may have a partial role, even though the better prognosis of $m E G F R$ patients has previously been attributed to mutational status. However, the literature lacks sufficient data to answer questions regarding the usefulness of adjuvant TKI in resected mEGFR patients. Indeed, the BR19 phase III study, which randomized 503 completely resected NSCLC patients (stages IB, II and IIIA) between adjuvant gefitinib and placebo, did not conclude that there was any benefit of adjuvant EGFR TKI treatment (HR: 0.57 (95\% CI: 0.14-2.33; Goss et al, 2013). However, that particular study suffered from several limitations as follows: (1) the treatment period differed between the placebo and the control group (8.9 and 4.8 months, respectively) and was shorter than periods usually observed in randomised study, and (2) the population was unselected; as a result, only $3 \%$ of the patients harboured an EGFR mutation. However, in a retrospective study from the Memorial Sloan Kettering Cancer Center on 167 completely resected mEGFR NSCLC patients ( $70 \%$ of stage IB, $15 \%$ of stage II and $15 \%$ of stage III), the authors concluded that with a median treatment period of 20 months, the use of adjuvant EGFR TKI was associated with a prolonged 2 -year DFS (89\% vs 72\%, HR: 0.53 (95\% CI: 0.28-1.03), $P=0.06$;
Janjigian et al, 2011). Consequently, ongoing trials may provide convincing evidence for customised therapies for resectable mEGFR NSCLC (Zhai et al, 2015).

One recent meta-analysis of 6939 patients concluded that $m K R A S$ was associated with decreased OS in NSCLC patients (HR: 1.45 (1.29-1.62)) (Meng et al, 2013). However, the main limitation of this study was the use of different chemotherapy regimens across studies. Our data are consistent with this meta-analysis because $m K R A S$ was associated with reduced OS. Specific $m K R A S$ mutations have been identified in lung cancer based on amino-acid substitution (Bamford et al, 2004), and it was found that individual $m K R A S$ mutations affect downstream signalling in different ways. Therefore, both KRAS G12C and G12V exhibited activated Ral signalling and decreased growth factor-dependent Akt activation, although the G12D mutation exhibited activated PI3K and MEK signalling (Ihle et al, 2012). Therefore, distinct amino-acid substitutions might activate different signalling pathways, which can lead to unique responses to chemotherapy and different clinical behaviours. Garassino et al (2011) demonstrated the association of KRAS G12C with a reduced response to cisplatin and increased sensitivity to Taxol and pemetrexed in NSCLC cell lines, whereas the G12V mutation was more resistant to pemetrexed. However, the clinical data are in conflict. In a study of 85 patients, Nadal et al (2014) demonstrated an association between KRAS G12C and worsening OS and DFS in 85 patients with resected NSCLC. In contrast, Izar et al (2014) identified improved OS and DFS for G12C and G12V patients in 127 patients. However, these two studies were based on small populations, hence the limited amount of data forbids use from drawing conclusions. To our knowledge, the cohort studied here represents the largest evaluation of the prognostic value of KRAS according to aminoacid substitution in resected NSCLC. Our observations that $m K R A S$ patients experienced worse OS and DFS are consistent with the literature. However, OS and TTR rates differed according to the type of amino-acid substitution. For example, the OS reached 62 months for G12R patients and 60 months for G12S patients. In contrast, the $\mathrm{G} 12 \mathrm{~V}$ mutation was associated with worse OS (decreasing to 26 months) and TTR (only 12 months). Furthermore, comparison of the G12V patients to the entire cohort revealed that the G12V mutation remained the only independent prognostic factor for TTR and recurrence and was associated with angioinvasion for OS. Recently, Alamo Alamo et al (2015) demonstrated that G12V mutants exhibited a high percentage of C-X-C chemokine receptor type 4 (CXCR4) in a colorectal cancer model. The CXCR4 is a highly conserved G protein-coupled receptor that binds at its CXCL12 ligand (a chemoattractant molecule) (Romain et al, 2014). CXCL12 is constitutively expressed in blood vessels, liver or lymph nodes (Romain et al, 2014). The overexpression of CXCR4 on colorectal cancer G12V cell lines suggests that this factor may also be found in NSCLC. This overexpression may partially explain the increased aggressiveness of tumours in G12V patients, that is, such patients exhibit higher chemoattraction.

It is important to note that our study suffers from several limitations, which we further consider here. Our study was a retrospective cohort study from a single treatment centre. The studied population was highly heterogeneous with different stages of disease and received different neo-adjuvant and adjuvant chemotherapy regimens. mKRAS patients exhibited the most aggressive disease and high pTNM and were more likely to benefit from adjuvant and neo-adjuvant chemotherapy. Therefore, due to the low number of mKRAS patients remaining $(n=30)$ in the cohort, we were unable to perform an analysis that focused on patients who had not undergone peri-operative treatment. However, even if peri-operative treatment and pTNM were prognostic factors of OS in univariate analysis, these factors were not significant in multivariate analysis. 
In conclusion, our study supports the hypothesis that EGFR and KRAS mutations are prognostic biomarkers in resected NSCLC. $m E G F R$ was likely associated with improved OS compared with WT and $m K R A S$, but the role of TKIs on this improved course cannot be excluded. To our knowledge, our report represents the largest data set on the prognosis of specific KRAS amino-acid substitutions in resected NSCLC. Notably, KRAS G12V was associated with a worse prognosis, supporting the molecular explanation. KRAS and EGFR may help patients adapt to adjuvant treatment in resected NSCLC, regardless of the pTNM. Ongoing trials could help specify the role of adjuvant TKIs in mEGFR patients. However, our results must be interpreted with caution because of the limitations listed above. Prospective multicentre cohort studies are mandatory to confirm these preliminary results.

\section{ACKNOWLEDGEMENTS}

We acknowledge the CRB (Centre de Ressources Biologiques), Biological Resource Centre, Strasbourg, France.

\section{CONFLICT OF INTEREST}

The authors declare no conflict of interest.

\section{REFERENCES}

Alamo P, Gallardo A, Di Nicolantonio F, Pavon MA, Casanova I, Trias M, Mangues MA, Lopez-Pousa A, Villaverde A, Vazquez E, Bardelli A, Cespedes MV, Mangues R (2015) Higher metastatic efficiency of KRas G12V than KRas G13D in a colorectal cancer model. Faseb J 29(2): 464-476.

Bamford S, Dawson E, Forbes S, Clements J, Pettett R, Dogan A, Flanagan A, Teague J, Futreal PA, Stratton MR, Wooster R (2004) The COSMIC (Catalogue of Somatic Mutations in Cancer) database and website. Br J Cancer 91(2): 355-358.

Beau-Faller M, Blons H, Domerg C, Gajda D, Richard N, Escande F, Solassol J, Denis MG, Cayre A, Nanni-Metellus I, Olschwang S, Lizard S, Piard F, Pretet JL, de Fraipont F, Bieche I, de Cremoux P, Rouquette I, Bringuier PP, Mosser J, Legrain M, Voegeli AC, Saulnier P, Morin F, Pignon JP, Zalcman G, Cadranel J (2013) A multicenter blinded study evaluating EGFR and KRAS mutation testing methods in the clinical nonsmall cell lung cancer setting-IFCT/ERMETIC2 project part 1: comparison of testing methods in 20 French Molecular Genetic National Cancer Institute platforms. J Mol Diagn 16(1): 45-55.

Beau-Faller M, Legrain M, Voegeli AC, Guerin E, Lavaux T, Ruppert AM, Neuville A, Massard G, Wihlm JM, Quoix E, Oudet P, Gaub MP (2009) Detection of K-Ras mutations in tumour samples of patients with non-small cell lung cancer using PNA-mediated PCR clamping. Br J Cancer 100(6): 985-992.

Bossard N, Velten M, Remontet L, Belot A, Maarouf N, Bouvier AM, Guizard AV, Tretarre B, Launoy G, Colonna M, Danzon A, Molinie F, Troussard X, Bourdon-Raverdy N, Carli PM, Jaffre A, Bessaguet C, Sauleau E, Schvartz C, Arveux P, Maynadie M, Grosclaude P, Esteve J, Faivre J (2007) Survival of cancer patients in France: a population-based study from The Association of the French Cancer Registries (FRANCIM). Eur J Cancer 43(1): 149-160.

Charlson ME, Pompei P, Ales KL, MacKenzie CR (1987) A new method of classifying prognostic comorbidity in longitudinal studies: development and validation. J Chronic Dis 40(5): 373-383.

D'Angelo SP, Janjigian YY, Ahye N, Riely GJ, Chaft JE, Sima CS, Shen R, Zheng J, Dycoco J, Kris MG, Zakowski MF, Ladanyi M, Rusch V, Azzoli CG (2012) Distinct clinical course of EGFR-mutant resected lung cancers: results of testing of 1118 surgical specimens and effects of adjuvant gefitinib and erlotinib. J Thorac Oncol 7(12): 1815-1822.
Garassino MC MM, Rusconi P, Rulli E, Martelli O, Farina G, Scanni A, Broggini M (2011) Different types of K-Ras mutations could affect drug sensitivity and tumor behavior in non-small-cell lung cancer. Ann Oncol 22(1): 235-237.

Goss GD, O'Callaghan C, Lorimer I, Tsao MS, Masters GA, Jett J, Edelman MJ, Lilenbaum R, Choy H, Khuri F, Pisters K, Gandara D, Kernstine K, Butts C, Noble J, Hensing TA, Rowland K, Schiller J, Ding K, Shepherd FA (2013) Gefitinib versus placebo in completely resected non-small-cell lung cancer: results of the NCIC CTG BR19 study. J Clin Oncol 31(27): 3320-3326.

Ihle NT, Byers LA, Kim ES, Saintigny P, Lee JJ, Blumenschein GR, Tsao A, Liu S, Larsen JE, Wang J, Diao L, Coombes KR, Chen L, Zhang S, Abdelmelek MF, Tang X, Papadimitrakopoulou V, Minna JD, Lippman SM, Hong WK, Herbst RS, Wistuba, Heymach JV, Powis G (2012) Effect of KRAS oncogene substitutions on protein behavior: implications for signaling and clinical outcome. J Natl Cancer Inst 104(3): 228-239.

Izar B, Zhou H, Heist RS, Azzoli CG, Muzikansky A, Scribner EE, Bernardo LA, Dias-Santagata D, Iafrate AJ, Lanuti M (2014) The prognostic impact of KRAS, its codon and amino acid specific mutations, on survival in resected stage I lung adenocarcinoma. J Thorac Oncol 9(9): 1363-1369.

Janjigian YY, Park BJ, Zakowski MF, Ladanyi M, Pao W, D’Angelo SP, Kris MG, Shen R, Zheng J, Azzoli CG (2011) Impact on disease-free survival of adjuvant erlotinib or gefitinib in patients with resected lung adenocarcinomas that harbor EGFR mutations. J Thorac Oncol 6(3): 569-575.

Jemal A, Bray F, Center MM, Ferlay J, Ward E, Forman D (2011) Global cancer statistics. CA Cancer J Clin 61(2): 69-90.

Marks JL, Broderick S, Zhou Q, Chitale D, Li AR, Zakowski MF, Kris MG, Rusch VW, Azzoli CG, Seshan VE, Ladanyi M, Pao W (2008) Prognostic and therapeutic implications of EGFR and KRAS mutations in resected lung adenocarcinoma. $J$ Thorac Oncol 3(2): 111-116.

Meng D, Yuan M, Li X, Chen L, Yang J, Zhao X, Ma W, Xin J (2013) Prognostic value of K-RAS mutations in patients with non-small cell lung cancer: a systematic review with meta-analysis. Lung Cancer 81(1): $1-10$.

Nadal E, Chen G, Prensner JR, Shiratsuchi H, Sam C, Zhao L, Kalemkerian GP, Brenner D, Lin J, Reddy RM, Chang AC, Capella G, Cardenal F, Beer DG, Ramnath N (2014) KRAS-G12C mutation is associated with poor outcome in surgically resected lung adenocarcinoma. J Thorac Oncol 9(10): 1513-1522.

Romain B, Hachet-Haas M, Rohr S, Brigand C, Galzi JL, Gaub MP, Pencreach E, Guenot D (2014) Hypoxia differentially regulated CXCR4 and CXCR7 signaling in colon cancer. Mol Cancer 13: 58.

Singh A, Greninger P, Rhodes D, Koopman L, Violette S, Bardeesy N, Settleman J (2009) A gene expression signature associated with "K-Ras addiction" reveals regulators of EMT and tumor cell survival. Cancer Cell 15(6): 489-500.

Thomas P, Dahan M, Riquet M, Massart G, Falcoz PE, Brouchet L, Le Pimpec Barthes F, Doddoli C, Martinod E, Fadel E. Porte Pour La Societe Francaise De Chirurgie Thoracique Et Cardio-Vasculaire H (2008) [Practical issues in the surgical treatment of non-small cell lung cancer. Recommendations from the French Society of Thoracic and Cardiovascular Surgery]. Rev Mal Respir 25(8): 1031-1036.

Uramoto H, Tanaka F (2014) Recurrence after surgery in patients with NSCLC. Transl Lung Cancer Res 3(4): 242-249.

Zhai H, Zhong W, Yang X, Wu YL (2015) Neoadjuvant and adjuvant epidermal growth factor receptor tyrosine kinase inhibitor (EGFR-TKI) therapy for lung cancer. Transl Lung Cancer Res 4(1): 82-93.

Zhang Z, Wang T, Zhang J, Cai X, Pan C, Long Y, Chen J, Zhou C, Yin X (2014) Prognostic value of epidermal growth factor receptor mutations in resected non-small cell lung cancer: a systematic review with metaanalysis. PLoS One 9(8): e106053.

This work is published under the standard license to publish agreement. After 12 months the work will become freely available and the license terms will switch to a Creative Commons AttributionNonCommercial-Share Alike 4.0 Unported License. 